\title{
Is the edge states energy spectrum of a 2D topological insulator linear?
}

(C) M.V. Entin ${ }^{1,2}$, M.M. Mahmoodian ${ }^{1,2, \uparrow, ~ L . I . ~ M a g a r i l l ~}{ }^{1,2}$

${ }^{1}$ Rzhanov Institute of Semiconductor Physics, Siberian Branch of the Russian Academy of Sciences, 630090 Novosibirsk, Russia

${ }^{2}$ Novosibirsk State University,

630090 Novosibirsk, Russia

I E-mail: mahmood@isp.nsc.ru

The linearity of the edge states spectrum in a 2D topological insulator, important for various transport phenomena, is studied. Different edge state models are examined. It is found that, in some of them, the linearity is perfect, while, in others, the linearity is approximate.

\section{Acknowledgements}

This research was supported by RSF grant No 17-02-01039. 